\title{
DESENVOLVIMENTO ORIENTADO POR HIPÓTESES: COMO AS LEAN STARTUPS CRIAM, EXPERIMENTAM E VALIDAM AS SUAS HIPÓTESES DE NEGÓCIO
}

Marcelo Henrique Gomes Couto ${ }^{1}$

Marcus Vinícius Higino Maida ${ }^{2}$

Alvair Silveira Torres Junior ${ }^{1}$

Fábio Lotti Oliva ${ }^{1}$ 


\section{DESENVOLVIMENTO ORIENTADO POR HIPÓTESES: COMO AS LEAN STARTUPS CRIAM, EXPERIMENTAM E VALIDAM AS SUAS HIPÓTESES DE NEGÓCIO}

Resumo: O presente artigo busca investigar e descrever como as lean startups criam, experimentam e validam as suas hipóteses nas duas primeiras etapas da abordagem do customer development. Para o cumprimento do objetivo proposto, adota-se o método de múltiplos casos, a abordagem qualitativa e o caráter exploratório-descritivo. Como estratégia de coleta dos dados utiliza-se a entrevista semiestruturada, analisada posteriormente por meio do método de análise de conteúdo. Amplamente utilizada, a abordagem do customer development apresenta-se como um guia sistemático de orientação flexível o suficiente para ser adaptado ao contexto no qual o negócio desenvolve-se, bem como aos recursos e condições disponíveis para o desenho e execução do processo. Entretanto os resultados sugerem que negócios que buscam desenvolver soluções mais disruptivas ou que se desenvolvem em mercados altamente regulamentados podem apresentar um desenvolvimento menos estruturado quando comparado ao processo sistemático proposto pela abordagem.

Palavras-chave: Lean startup. Customer development. Estratégia orientada por hipóteses. Experimentação.

\section{Introdução}

A incerteza sempre foi uma característica do ambiente de negócios e um fator associado ao processo de atendimento a uma oportunidade (Janeway, 2012; Sull, 2004). Entretanto o seu grau parece aumentar de maneira crescente à medida que a economia global avança e experimenta mudanças tecnológicas mais rápidas (Teece, Peteraf \& Leih, 2016), o que por sua vez imprime novos desafios inexplorados aos empreendedores e exige transformações dinâmicas em seus modelos de negócio (Blank, 2013; Ries, 2012; Teece et al., 2016).

Assim, frente a essas constantes pressões e tensões causadas pela dinâmica global, os princípios da administração tradicional que ora promoveram o sucesso das organizações no último século, parecem agora mal adaptados para lidar com tal contexto de incerteza (Ries, 2012) e, apesar de inexistentes mecanismos de mercado que protejam a inovação empresarial dessas incertezas (Janeway, 2012), a agilidade tem se apresentado como uma capacidade organizacional valiosa a ser desenvolvida pelas organizações modernas que buscam pelo desenvolvimento sustentado (Ries, 2012; Teece et al., 2016). Não por acaso, as empresas que atualmente têm alcançado o sucesso são de fato aquelas que além de atender às demandas latentes no tempo certo, também são rápidas e flexíveis o suficiente em termos de capacidade inovativa (Teece, Pisano \& Shuen, 1997).

Desta forma, é nesse contexto que um método relativamente recente e promissor e que promete ainda aumentar a agilidade e diminuir os riscos no processo de desenvolvimento de novos negócios em ambientes de incerteza tem emergido e sido amplamente empregado pelos empreendedores (Blank, 2013; Ries, 2012; Teece et al. , 2016; York \& Danes, 2014).

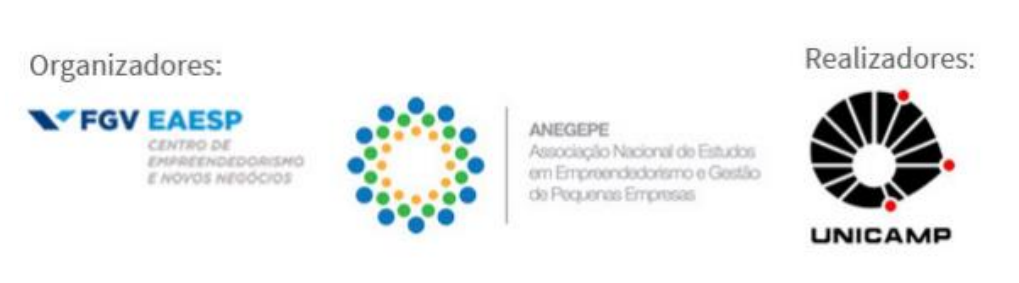


A partir de um processo estratégico orientado por hipóteses (Blank, 2013; Eisenmann, Ries \& Dillard, 2013; Ries, 2012), o lean startup busca além de reduzir os riscos, tempo e consumo de recursos, eliminar os inúmeros desperdícios que podem envolver o processo de desenvolvimento de um novo negócio, por meio da aplicação de uma abordagem que se sustenta sobre a aplicação dos princípios do pensamento enxuto ao processo de inovação, encorajando uma abordagem mais científica para a tomada de decisão e a participação dos clientes e usuários durante todo o processo de desenvolvimento da solução (Bieraugel, 2015; Blank, 2013; Ries, 2012).

Conhecida como customer development, essa abordagem na qual o método lean startup sustenta-se é caracterizada, portanto, por um processo sistemático e iterativo que organiza a busca por um modelo de negócio em que, a partir do feedback dos clientes, uma startup pode descobrir se as hipóteses de negócio previamente criadas pelo empreendedor são válidas mercadologicamente e, em caso de invalidade, pivotar para novas hipóteses ou mesmo abandonar a ideia (Blank, 2013; Eisenmann et al., 2013).

Assim, enquanto a criação das hipóteses preocupa-se com a forma como o empreendedor inicialmente julga ser "verdadeira" sua suposição, o teste busca fornecer dados e informações confiáveis para a tomada de decisão quanto à validação para condução do negócio (Blank \& Dorf, 2014; Ries, 2012; York \& Danes, 2014). Contudo apesar de promover a experimentação científica para descobrir como desenvolver um negócio inicial, o processo de validação das hipóteses que compõem a abordagem não possui um sentido tradicional em si, uma vez que o empreendedor formula e avalia suas suposições com base em julgamentos e decisões baseadas no pensamento intuitivo e no contato direto com o cliente ou usuário, o que torna esse processo propenso a vieses e erros (York \& Danes, 2014).

Deste modo, visto que a experimentação é um processo fortemente estimulado ao longo da abordagem do customer development, pesquisas adicionais sobre o design proposto pela abordagem - e consequentemente sobre os experimentos por ela estimulados - faz-se necessário, visto que podem contribuir para uma melhor compreensão da abordagem em sua completude (Frederiksen \& Brem, 2016), assim como dos possíveis erros e vieses que podem estar envolvidos na sua execução (York \& Danes, 2014).

Portanto, o presente artigo busca investigar e descrever como as lean startups criam, experimentam e validam as suas hipóteses nas duas primeiras etapas da abordagem do customer development, proposta por Blank (2013).

\section{Referencial Teórico}

\subsection{Lean Startups}

Desenvolvidos inicialmente na Toyota por Taichii Ohno e Shigeo Shingo, os princípios do desenvolvimento lean foram usados a priori para melhorar a eficiência dos processos de fabricação e reduzir os desperdícios na companhia (Bieraugel, 2015; Ries, 2012; Ward \& Sobek, 2014), determinando quais partes do processo eram capazes de agregar valor para o cliente e quais partes não eram, o que se caracterizava então como um desperdício (Frederiksen \& Brem, 2016). Assim, apesar de na época do desenvolvimento o conjunto de princípios aparentar presumivelmente ineficiência, sua adoção pela companhia resultou, entretanto, num sistema geral mais eficiente que, a partir de um conjunto mais amplo de projetos possíveis e
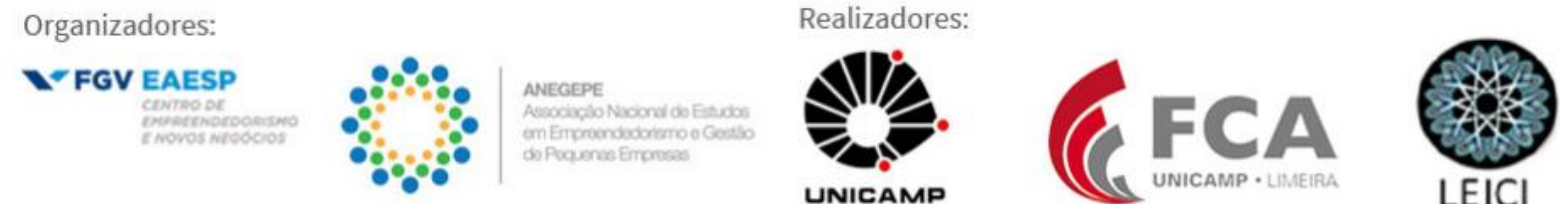
atraso em certas tomadas de decisão, gerou ciclos mais rápidos de desenvolvimento de veículos que os demais concorrentes (Sobek II, Ward \& Liker, 1999).

Desde então, após comprovada a eficiência dos princípios visto os resultados promovidos no desempenho da Toyota (Sobek II, Ward \& Liker, 1999) e motivadas pela busca de novos métodos e tecnologias mais eficientes em termos de custo, tempo e qualidade (AlAshaab, Golob, Urrutia, Gourdin, Petritsch, Summers \& El-Nounu, 2015; Edison et al., 2018), a filosofia lean sido usada para remover o desperdício de tempo ou de etapas desnecessárias em diferentes processos no ambiente de negócios (Bieraugel, 2015), alterando fortemente a maneira pela qual os sistemas de produção são conduzidos nas empresas (Ries, 2012).

Assim, buscando eliminar os inúmeros desperdícios que podem envolver o processo de desenvolvimento de uma startup, Ries (2012) aplica então os princípios do pensamento enxuto ao processo de inovação em negócios e o denomina de lean startup, apresentando-o como um método baseado em um conjunto de processos para reduzir tempo, dinheiro e risco ao iniciar uma nova empresa (Bieraugel, 2015; Ries, 2012). Além disso, sustentado sobre uma abordagem orientada a uma experimentação mais científica para descobrir como desenvolver um negócio sustentável em um ambiente de extrema incerteza (Ries, 2012), Ries apresenta ainda cinco princípios de sustentação que são, no geral, capazes de acelerar o ciclo de desenvolvimento do negócio (Bieraugel, 2015; Blank, 2013; Ries, 2012) e generalizara a aplicação do método em diversos tipos de negócios (Ries, 2012).

Desta forma, uma lean startup pode ser então entendida como um novo empreendimento que orienta o seu desenvolvimento por meio de hipóteses que devem ser testadas para avaliar uma oportunidade de negócio a fim de desenvolver um novo produto ou serviço para um determinado nicho de mercado (Eisenmann et al., 2013) e que busca reduzir a incerteza extrema associada em si ao envolver o cliente ao longo de seu desenvolvimento e utilizar o teste incremental das inovações (Bieraugel, 2015; Blank, 2013; Ries, 2012).

Sob essa perspectiva, para Ries (2012) o processo de desenvolvimento de uma lean startup inicia-se com o estabelecimento de uma visão e, posteriormente, decomposição dessa visão em suposições que o mesmo denomina de hipóteses. Depois disso, o processo é conduzido pela capacidade da startup em perseverar ou pivotar de maneira rápida as hipóteses de negócio formuladas, testando, descartando e substituindo ideias ou modelos que não funcionam (Blank, 2013; Ries, 2012; Teece et al., 2016).

Entretanto esse processo não é simples e para alcançar os resultados de uma experimentação são necessários procedimentos e técnicas. Neste sentido, uma técnica que Ries (2012) denomina de Mínimo Produto Viável (MVP) pode ser utilizada como meio de testar uma hipótese. Contudo diferentemente de um protótipo ou teste de conceito, um MVP é projetado não só para responder a perguntas técnicas ou de design. Ele também ajuda os empreendedores a começar o processo de aprendizagem validada de maneira mais rápida e com o menor esforço possível (Ries, 2012), utilizando-se de princípios ágeis como ferramentas para coletar feedback dos clientes sobre o produto através do uso da própria solução desenvolvida (Bieraugel, 2015; Blank, 2013; Edison et al., 2017).

Assim, à medida que os clientes interagem com o produto eles geram feedback e dados para as startups (Ries, 2012). Esses feedbacks podem ser tanto qualitativos, quanto quantitativos e constituem-se como uma importante fonte de informações para o aprendizado e, consequentemente, aprimoramento da solução e desenvolvimento da empresa (Blank, 2013;

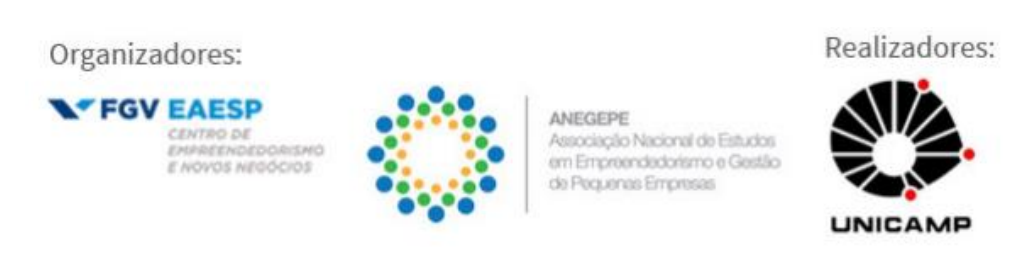


Blank \& Dorf, 2014; Frederiksen \& Brem, 2016; Ries, 2012). Denominado como Ciclo de Feedback Construir-Medir-Aprender, esse processo, portanto, é capaz de gerar um aprendizado que orienta onde e quando a startup deve investir energia, resultando em economia de tempo e dinheiro (Ries, 2012).

Logo, o desenvolvimento da aprendizagem validada por meio de hipóteses é conduzido pelos dados empíricos coletados a partir do processo iterativo e incremental com os clientes (Blank, 2013; Blank \& Dorf, 2014; Edison et al., 2017; Ries, 2012), que por sua vez são confrontados com os dados atuais da empresa, permitindo que marcos de aprendizagem sejam elaborados (Blank, 2013; Ries, 2012). Isso exige que os dados sejam rigorosamente medidos e avaliados por intermédio de métricas coerentes ao objetivo de mensuração, o que Ries (2012) identifica como métricas acionáveis.

Deste modo, cada estágio do processo como um todo é iterativo e uma startup poderá falhar algumas vezes antes de encontrar a abordagem correta (Blank, 2013; Sull, 2004). Por isso, a resposta às falhas pode ser crítica para que o processo associado à tentativa e erro desenvolva-se e, neste sentido, a capacidade de aprender com experiências erradas e a resiliência para continuar experimentando são capacidades individuais e organizacionais fundamentais para a evolução da startup (Sosna, Trevinyo-Rodríguez \& Velamuri, 2010; Sull, 2004).

\subsection{O desenvolvimento orientado por hipóteses: da criação a validação}

Para Ries (2012) a primeira etapa para entender uma solução é descobrir se ela cria ou destrói valor. Entretanto apesar de defender que para construir uma solução de maneira iterativa é necessário entregá-la ao mercado o mais rápido possível, no início de uma startup os clientes são geralmente desconhecidos e, consequentemente, o que podem perceber como valor também (Edison et al., 2017; Ries, 2012). Assim, para solucionar esta problemática uma startup deve construir uma estratégia que inclua o desenvolvimento de um modelo de negócio, um plano de produto e uma suposição a ser validada acerca dos parceiros e dos concorrentes, bem como de quem sejam os clientes (Ries, 2012).

Entretanto para que essa estratégia seja eficiente, os empreendedores devem identificar e entrar em contato com o mercado potencial ou hipotético o mais rápido possível, de modo a validar o problema que pretendem resolver e também descobrir quem são seus clientes na prática (Blank, 2013; Blank \& Dorf, 2014; Ries, 2012). Para isso, a ideia é que um MVP seja criado de maneira ágil, lançado e, a partir das iterações com o mercado, a startup possa aprender rapidamente com as informações obtidas a fim de ajustá-lo adequadamente e o melhorar (Teece et al., 2016).

Sob essa perspectiva, conhecida como customer development, a abordagem na qual o método lean startup baseia-se pode ser dividida em quatro etapas, na qual as duas primeiras focam-se prioritariamente na identificação do que agrega valor aos clientes e as duas últimas na criação de um mercado para o produto e dimensionamento do negócio (Blank, 2013; Edison et al., 2017). Isso ajuda os empreendedores a reduzir o maior risco e desperdício enfrentados por novos negócios: oferecer um produto que o mercado não deseja (Ries, 2012).

Contudo apesar desse processo relativamente simples muitas startups ainda falham devido ao desperdício de recursos na criação e comercialização de soluções antes mesmo que as incertezas sejam minimizadas para o desenvolvimento de um modelo de negócio (Blank,

\section{Organizadores:}

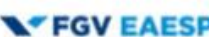

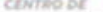

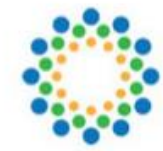

ANEGeBPE

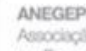

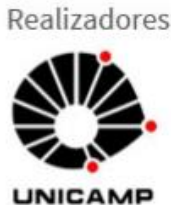

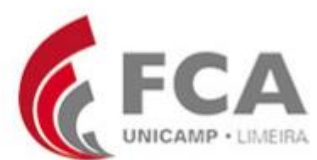

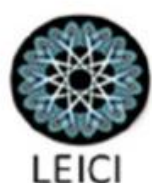


2013; Eisenmann et al., 2013; Sull, 2004). Assim, para que isso seja evitado além da orientação fornecida pela abordagem é necessário que o empreendedor desenvolva uma compreensão clara e objetiva do seu modelo de negócio e de quais elementos são mais importantes para o sucesso da empresa (Crews, 2016). Além disso, desenvolver uma abordagem inicial que direcione as hipóteses não para o crescimento, mas para o aprendizado sobre a construção de um negócio sustentável (Eisenmann et al., 2013) e um entendimento sobre como usar recursos limitados de forma mais eficiente (Ries, 2012; Sull, 2004) é também igualmente importante.

Logo, o processo para formular uma hipótese inicial deve ser caracterizado por um modelo esquemático que inclua a definição da oportunidade, os recursos necessários, o valor a ser criado e um plano para alcança-los (Sull, 2004). Nesse sentido, Blank (2013) fornece de maneira prática uma visão geral rápida do método lean startup com o suporte do modelo de negócio canvas para ajudar o empreendedor a definir suas hipóteses iniciais. No entanto o desenvolvimento de hipóteses generalistas, ou seja, baseadas apenas no modelo de negócio como um todo, não é suficientemente capaz de conduzir a uma produção eficiente de informações estratégicas e, por isso, identificar um elemento particular do modelo de negócios para dirigir o processo de experimentação de maneira gradual é fundamental (Crews, 2016).

Assim, de modo a orientar o processo de formulação de hipóteses em um negócio inicial, Ries (2012) sugere a decomposição da visão do negócio em duas suposições ou hipóteses distintas, denominadas por ele de hipóteses de valor e hipóteses de crescimento, que se diferenciam quanto à sua natureza e objetivos. Essas hipóteses seguem o raciocínio empregado por Blank (2013) no desenvolvimento orientado pelo customer development e complementam de igual maneira sugerem direções e esforços distintos nas etapas de desenvolvimento de uma startup.

Entretanto apesar de distintas quanto a sua função e objetivo, uma característica comum a elas e ao processo orientado por hipóteses como um todo é que ambos seguem as "intuições" iniciais do empreendedor em relação ao seu modelo de negócio, no qual o processo iterativo com clientes e usuários é fundamental para aquisição de conhecimento, teste e condução (Blank \& Dorf, 2014; York \& Danes, 2014). Desta forma, é possível reconhecer o papel da "intuição" dentro desse processo (York \& Danes, 2014), ainda que o mesmo envolva uma abordagem na qual a criação e avaliação de hipóteses exija determinado rigor (Frederiksen \& Brem, 2016).

Portanto, a geração de hipóteses é essencialmente um processo baseado na intuição do empreendedor sobre o mercado, começando com suposições intuitivas sobre a natureza do(s) problema(s) e os clientes que a empresa se propõe abordar (Blank, 2013). No entanto visto o ambiente no qual as startups estão inseridas, as premissas implícitas e explícitas sobre as múltiplas variáveis que compõem uma ou mais hipóteses iniciais podem ser amplamente incertas, dificultando a previsão e a modelagem do futuro pelo empreendedor (Sull, 2004). Mesmo assim, capacidades para criar hipóteses sobre implicações futuras de eventos e tendências de maneira rápida, ainda que em ambientes incertos, é fundamental para detectar reais oportunidades de negócio antes que estas se tornem evidentes para o mercado (Teece et al., 2016). E para isso, Teece et al. (2016) apresentam um conjunto de capacidades essenciais para alcançar esses resultados (Quadro 1).

\begin{tabular}{|l|l|}
\hline Capacidade & Descrição \\
\hline
\end{tabular}

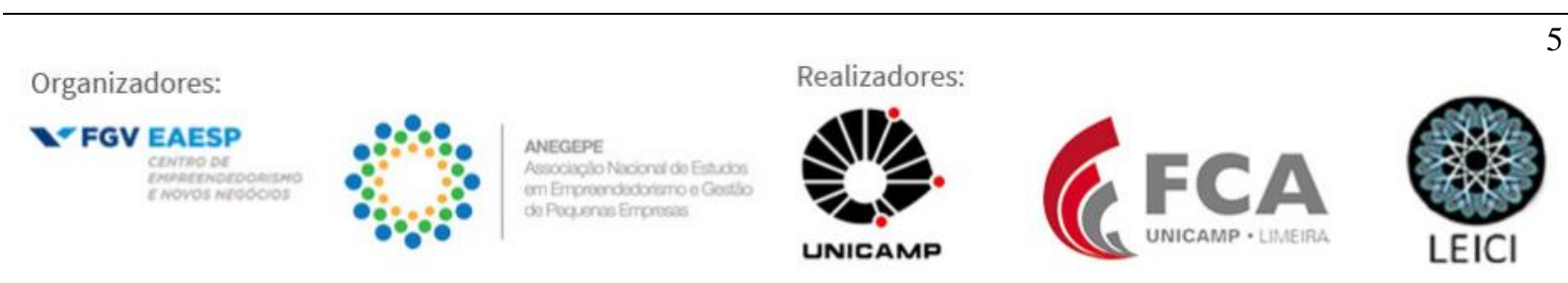




\begin{tabular}{|l|l|}
\hline $\begin{array}{l}\text { Detecção } \\
\text { generativa }\end{array}$ & $\begin{array}{l}\text { Em resumo, refere-se à capacidade de construir hipóteses e aprender, assistido } \\
\text { pela abdução. A abdução é um modo de inferência que utiliza todos os dados } \\
\text { disponíveis para identificar padrões coerentes e testar. }\end{array}$ \\
\hline $\begin{array}{l}\text { Planejamento de } \\
\text { cenários }\end{array}$ & $\begin{array}{l}\text { Ferramenta ou método que permite imaginar futuros possíveis em que decisões } \\
\text { organizacionais podem ser necessárias. }\end{array}$ \\
\hline Sensemaking & $\begin{array}{l}\text { É modo como as pessoas interpretam e a capacidade de atribuir sentido às novas } \\
\text { informações visando reduzir o grau de ambiguidade. }\end{array}$ \\
\hline $\begin{array}{l}\text { "Compra de } \\
\text { opções reais" }\end{array}$ & Capacidade de "investir" no momento certo e por um custo menor. \\
\hline
\end{tabular}

Quadro 1: Capacidades fundamentais para criar hipóteses sobre implicações futuras

Fonte: Adaptado de Teece et al. (2016).

Assim, os atributos descritos no Quadro 4 representam capacidades para sentir futuros e oportunidades emergentes e, neste sentido, desenvolver hipóteses a cerca dos mesmos para validação rápida, por meio da exploração e interpretação dos dados disponíveis e a identificação de padrões coerentes (Teece et al., 2016). Embora seja um movimento amplamente disseminado no ambiente de negócios atual, encontrar padrões em dados, contudo, não necessariamente traduz uma relação de causa e efeito, o que pode induzir à tomada de decisões equivocadas, visto que os reais motivos para a compra de uma solução são mais específicos e não apenas capturados por correlações entre padrões de dados (Christensen, Hall, Dillon, \& Duncan, 2016). Para solucionar este equívoco, Christensen et al. (2016) sugerem que as empresas devem utilizar o que chamam de job to be done.

Ainda neste sentido, Sull (2004) também sugere outro conjunto prescritivo de condições, na tentativa de minimizar a incerteza e prestar suporte a construção de hipóteses iniciais mais eficientes, mesmo antes do processo de experimentação em si (Quadro 2).

Flexibilidade inicial para adaptar as hipóteses. Uma hipótese provavelmente seja alterada, em maior ou menor grau, no primeiro contato com o mercado.

Exame, a priori, das hipóteses à luz das próprias experiências empreendedoras, de modo a triar inicialmente uma oportunidade hipotética. Para isso, é necessário um profundo conhecimento dos clientes, bem como dos fatores competitivos, técnicos e regulatórios do negócio. Isso possibilita uma avaliação da oportunidade antes da experimentação real.

Identificar variáveis que provavelmente serão fatais para o negócio, assim como variáveis que sejam impulsionadoras do sucesso.

Quadro 2: Prescrições para construção de hipóteses em ambientes de incerteza

Fonte: Adaptado de Sull (2004).

Conforme observado no Quadro 2, apesar da falta de experiência ou conhecimento empreendedor sugerir uma provável barreira no processo de formulação de hipóteses, ela pode não ser, no entanto, um fator limitante nesse processo. Segundo os estudos de McMullen \& Kier (2017), a imaginatividade (enquanto habilidade cognitiva) parece ser especialmente útil para indivíduos que não possuem experiência empresarial, permitindo que qualquer pessoa crie e selecione ideias de um novo negócio com a mesma proficiência de um empreendedor habitual.

Entretanto criar uma hipótese é apenas a primeira etapa do processo. Continuamente, depois de criadas as hipóteses, elas devem ser então testadas por meio de experimentação (Blank, 2013; Ries, 2012; Sosna et al., 2010; Sull, 2004; Teece et al., 2016). Neste sentido, no 
contexto empresarial um experimento é um teste de curto prazo e escala limitada, projetado para reduzir as fontes de incerteza e responder questões críticas para o sucesso antes que recursos adicionais sejam comprometidos (Sull, 2004; Ries, 2012; Euchner \& Ganguly, 2014). Deste modo, a partir dos princípios no qual o lean startup baseia-se, Ries (2012) sugere que os experimentos devem se valer de MVPs construídos de maneira rápida para apresentação junto aos clientes ou usuários onde o problema ou fenômeno hipotético a ser solucionado acontece e ser utilizado de maneira iterativa e incremental (Blank, 2013).

Assim, cada hipótese testada fornece um conjunto de informações e produz uma decisão, que por sua vez envolve outro conjunto de hipóteses que deve ser novamente testado e avaliado (Ries, 2012). Portanto, esse processo incremental e iterativo é quase que inteiramente derivado de informações subjetivas, obtidas por contato direto com potenciais clientes, fornecedores e parceiros (Blank, 2013). Isso, segundo York \& Danes (2014), pode ser classificado como um processo de tomada de decisão bayesiano, pois, desenvolve hipóteses baseadas em experiência ou intuição, identifica fontes de dados úteis para avaliá-las e seleciona um curso de ação com base nessa avaliação. Contudo ainda segundo York \& Danes (2014), essa forma de teste de hipóteses é informal e propensa a erros e vieses de julgamento que podem prejudicar sua precisão (Quadro 3).

\begin{tabular}{|l|l|}
\hline \multicolumn{1}{|c|}{ Viés } & \multicolumn{1}{c|}{ Descrição } \\
\hline De seleção & $\begin{array}{l}\text { Os dados são obtidos a partir de fontes de confirmação "amigáveis" resultando } \\
\text { em amostragem não representativa do(s) mercado(s) alvo. }\end{array}$ \\
\hline $\begin{array}{l}\text { De } \\
\text { representatividade }\end{array}$ & Generalizar a partir de pequenas amostras e dados não aleatórios. \\
\hline De aquiescência & $\begin{array}{l}\text { A tendência dos entrevistados de dar as respostas que o empreendedor deseja } \\
\text { ouvir. }\end{array}$ \\
\hline De confirmação & $\begin{array}{l}\text { Os entrevistados tendem a favorecer ou interpretar a informação de forma a } \\
\text { confirmar suas crenças anteriores. }\end{array}$ \\
\hline $\begin{array}{l}\text { De excesso de } \\
\text { confiança }\end{array}$ & $\begin{array}{l}\text { O excesso de confiança pode levar ao bloqueio de novas evidências ou } \\
\text { perspectivas alternativas, a partir da superestimação dos conhecimentos, } \\
\text { habilidades e precisão das informações. }\end{array}$ \\
\hline De otimismo & $\begin{array}{l}\text { A crença do empreendedor de que o mesmo provavelmente não experimentará } \\
\text { resultados negativos ou falhará. }\end{array}$ \\
\hline
\end{tabular}

Quadro 3: Erros e vieses de julgamento associados ao teste de hipóteses informal

Fonte: Adaptado de York \& Danes (2014).

Deste modo, diante dos erros e vieses de julgamento possíveis e descritos no Quadro 3, assim como acontece no próprio método cientifico em si, é também crucial neste processo informal além de isolar a hipótese e especificar como a mesma será testada, estabelecer o que será medido e como será interpretado (Euchner \& Ganguly, 2014). Neste sentido, Ries (2012) propõem o desenvolvimento do que cunhou de contabilidade para a inovação. Diferentemente da contabilidade tradicional, ela permite estabelecer o que e como devem ser medidos os esforços de ajuste, de modo a aumentar a garantia de que os resultados certos serão mensurados e avaliados, a fim de validar ou rejeitar as hipóteses (Ries, 2012; Frederiksen \& Brem, 2016) para a tomada de decisão quanto ao curso de ação de modo mais eficiente (Blank, 2013; Blank \& Dorf, 2014).
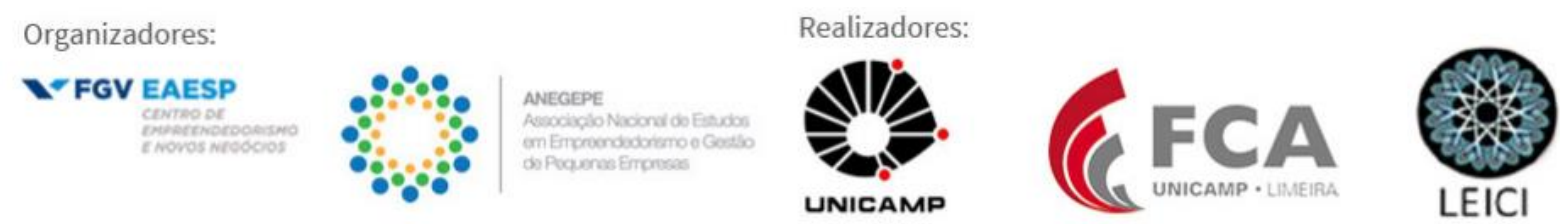


\section{Metodologia}

\subsection{Sujeitos de pesquisa}

Mediante o objetivo proposto, para compor o conjunto de sujeitos de análise seis startups foram selecionadas e entrevistadas. Assim, para o mapeamento e identificação das startups que compuseram os sujeitos de análise, além das redes de contato dos pesquisadores utilizou-se também duas premissas básicas: i) a utilização da orientação por hipóteses como abordagem de desenvolvimento pela startup e; ii) estar nas etapas de processo de descoberta e validação pelo cliente. Contudo apesar das seis startups entrevistadas, apenas quatro compuseram o sujeito final de pesquisa, visto à qualidade e saturação das informações alcançadas. Todos os entrevistados além de fundadores ocupam também o cargo de CEO das startups estudadas. Isso assegura que o entrevistado detenha do conhecimento da trajetória da empresa, elemento essencial para a presente investigação.

\subsection{Delineamento de pesquisa}

O presente artigo adota a abordagem qualitativa de pesquisa, uma vez que essa abordagem se compõe de um conjunto de técnicas interpretativas que objetivam compreender e interpretar um determinado fenômeno (Cooper \& Schindler, 2016).

Quanto ao método, adota-se o estudo de casos múltiplos orientado sob a perspectiva proposta por Yin (2015), uma vez que além de responder a perguntas de como o fenômeno ocorre em um determinado ambiente, ao buscar uma investigação empírica e em profundidade a partir de múltiplas evidências, este método permite também compreender o fenômeno em questão de maneira mais exaustiva.

Por fim, este estudo classificou-se ainda como exploratório-descrito. Exploratório, pois, busca desenvolver uma melhor compreensão sobre um fenômeno ainda pouco explorado sob a perspectiva proposta e, descritivo, pois identifica e descreve um evento ou atividade (Hair, Babin, Money \& Samouel, 2005).

\subsection{Técnica de coleta de dados}

Como técnica de coleta de dados escolheu-se a entrevista semiestrutura (Cooper \& Schindler, 2015), na qual o roteiro de questões baseou-se principalmente nas definições constitutivas apresentados no Quadro 4.

\begin{tabular}{|l|l|}
\hline \multicolumn{1}{|c|}{ Termos } & \multicolumn{1}{c|}{ Definição constitutiva } \\
\hline Lean startup & Eisenmann et al. (2013). \\
\hline Customer development & Blank (2013) e Blank \& Dorf (2014). \\
\hline Hipótese & Ries (2012), Blank \& Dorf (2014) e York \& Danes (2014). \\
\hline Experimento & Sull (2004), Ries (2012) e Euchner \& Ganguly (2014). \\
\hline Viés de validação & York \& Danes (2014). \\
\hline Métricas & Ries (2012). \\
\hline
\end{tabular}

Quadro 4: Definições constitutivas adotadas no estudo

Fonte: Elaborado pelo autor (2017).
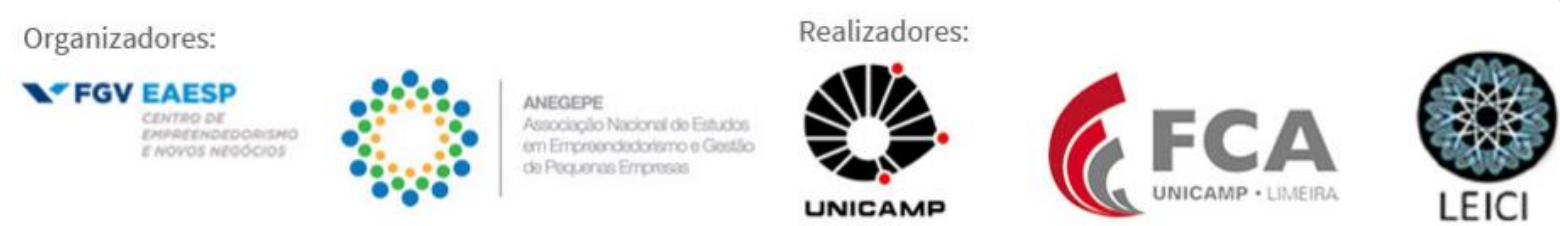
Além disso, o roteiro foi composto por 25 questões organizadas em quatro diferentes seções, a saber: i) identificação do respondente e da startup; ii) compreensão do desenvolvimento do negócio e da trajetória da startup até o momento atual; iii) identificação e compreensão do processo de criação e seleção de hipóteses; iv) identificação e compreensão do processo de validação das hipóteses e do aprendizado. Esta estruturação obedeceu, portanto, à organização lógica do processo estudado, bem como ao atendimento do objetivo proposto.

\subsection{Coleta, tratamento e análise dos dados}

A coleta dos dados aconteceu de maneira presencial ou via Skype ${ }^{\circledR}$ entre os dias $11 \mathrm{e}$ 15 do mês de dezembro de 2017. O tempo médio de duração das entrevistas foi de 50 minutos e os conteúdos obtidos foram registrados e armazenados em gravação digital e em anotações manuscritas, quando conveniente.

Assim, para análise dos conteúdos registrados, adotou-se o método de análise de conteúdo proposto por Bardin (2010) visto que, segundo a própria autora, este método possui um conjunto técnicas de análise de comunicação que por meio de procedimentos sistemáticos permite a descrição e análise do conteúdo das mensagens registradas. Além disso, a técnica escolhida desenvolveu-se sob as três etapas sequenciais propostas por Bardin (2010). Entretanto para início do processo proposto pela técnica, os conteúdos obtidos foram transcritos em programa de edição de textos.

Depois de transcritos, o processo de análise iniciou-se pela etapa de organização do conteúdo. A partir de uma leitura flutuante inicial das mensagens transcritas, os conteúdos que não atendiam o escopo do objetivo de pesquisa foram eliminados, assim como aqueles que o atendiam foram organizados e separados conforme o respectivo bloco estrutural do roteiro de entrevista a qual pertence. Logo, o próprio bloco apresentado e construído para organização do roteiro foi utilizado como forma de classificação e agregação do conteúdo ao contexto do objetivo, permitindo a execução das etapas de codificação e categorização.

Por fim, depois de categorizados e organizados os conteúdos selecionados foram interpretados de modo a atender a última etapa do processo: tratamento, inferência e interpretação dos resultados. Desta forma, desenvolveu-se a priori o tratamento dos conteúdos de cada caso em particular e posterior análise cruzada dos casos.

\section{Análise dos resultados}

\subsection{Caso 1}

A proposta de valor inicial da startup não sofreu nenhuma grande mudança estratégica desde a sua concepção. Em outras palavras, a startup A não efetuou nenhum movimento de pivô. No entanto otimizações da solução são frequentemente hipotetizadas e executadas, de modo a ajustar as funcionalidades às necessidades dos clientes e usuários, bem como responder às implicações futuras de tendências imaginadas e observadas pelos sócios.

Deste modo, assim como a hipótese de proposta de valor inicial, as demais hipóteses desenvolvidas são fundamentalmente criadas a partir da interação com cliente ou usuário no local onde o problema acontece (Blank, 2013; Ries, 2012), da observação e compreensão do problema em suas partes (Christensen et al., 2016) e da interpretação de eventos e tendências futuros (Teece et al., 2016). Ainda neste caso, a experiência empreendedora, o conhecimento
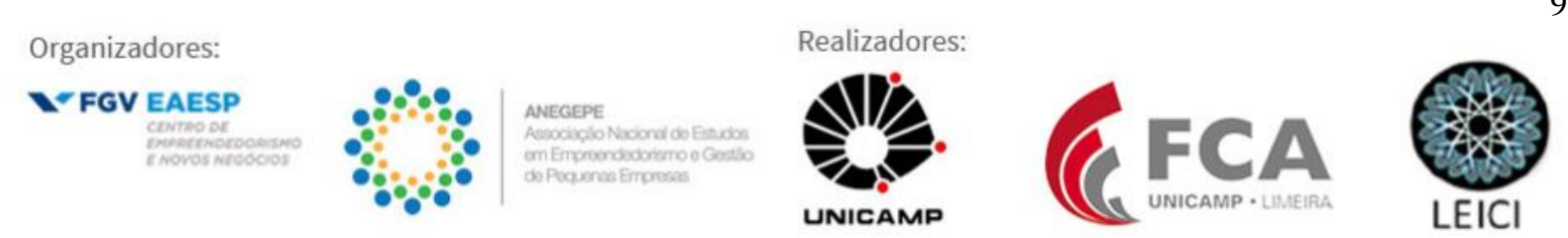
do setor e do cliente, bem como dos fatores competitivos, técnicos e regulatórios relacionados ao negócio é também importante para o desenvolvimento e seleção das hipóteses (Sull, 2004). Outras considerações para a criação e desenvolvimento das hipóteses de valor neste caso são a diferenciação clara de quem são os clientes e quem são os usuários da solução e a consequente compreensão e tradução do que representa valor para ambos (Ries, 2012).

No que se refere ao desenho do experimento e ao processo de validação das hipóteses, a startup A diferentemente do proposto pelo customer development opta por experimentar um conjunto de $n$ hipóteses de maneira simultânea, como forma de otimizar a velocidade do processo de desenvolvimento. Contudo esse processo somente é possível, pois, é suportado por uma organização descentralizada do trabalho e uma autonomia das partes terceirizadas de operação do projeto, o que permite desenvolver sistemas mais flexíveis e com maior capacidade de resposta (Teece et al., 2016). Todavia da figura de um coordenador de projetos que se assemelha à figura de um Shuza se torna essencial (Ward et al., 1995; Ward \& Sobek, 2014).

Assim, na prática as experimentações utilizam da própria solução e da iteração com o cliente como forma de teste. No entanto o teste não é utilizado apenas como uma fonte de obtenção de informações para validação de hipóteses, mas também como uma oportunidade de oferecer uma experiência ao usuário (Christensen et al., 2016). Para isso, contudo, é necessário que a empresa possua um conhecimento profundo dos clientes e do negócio (Sull, 2004), assim como do job to be done do problema em foco (Christensen et al., 2016).

Por fim, a validação das hipóteses é realizada pelo CEO e, assim como proposto na abordagem de Blank, acontece principalmente com base no feedback do cliente, atendendo a uma maioridade percentual de aceitação ou rejeição do valor proposto, obtida a partir de um número mínimo arbitrário de $n$ clientes ou usuários avaliados. Além disso, após a experimentação os aprendizados são armazenados digitalmente junto aos resultados do experimento e às métricas utilizadas para tomada de decisão daquele experimento.

\subsection{Caso 2}

Desde a sua criação, a visão inicial da startup B sofreu apenas uma grande mudança estratégica, caracterizando um movimento de pivô desenvolvido não como resposta a uma invalidação de uma hipótese, mas como uma mudança estruturada e projetada para testar uma nova hipótese fundamental acerca da solução e do modelo de negócios capaz de potencializar o crescimento da empresa (Ries, 2012) e, consequentemente, ser mais exponencial e lucrativa (Blank \& Dorf, 2014).

Deste modo, assim como a hipótese inicial do negócio, as hipóteses sequenciais são criadas, a priori, como suposições que o fundador julga serem verdadeiras (Blank \& Dorf, 2014; York \& Danes, 2014; Ries, 2012), obtidas em grande parte pela capacidade de integrar a imaginatividade, enquanto habilidade cognitiva que combina a imaginação com o conhecimento necessário para aplicar essa imaginação à ideação do empreendimento (McMullen \& Kier, 2017), ao processo de interpretar e atribuir sentido às informações percebidas pelo CEO para reduzir a incerteza (Teece et al., 2016). Além disso, destaca-se a habilidade demonstrada pelo CEO de observar, identificar e integrar soluções e tecnologias desenvolvidas e validadas em outros diferentes modelos de negócio, a fim de se investir em hipóteses de valor já validadas pelo mercado (Teece et al., 2016) e reduzir o consumo de

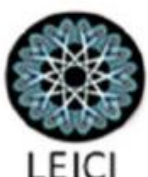


recursos e tempo. O feedback dos clientes (Blank, 2013; Ries, 2012), por fim, completa as fontes de obtenção das hipóteses neste caso.

Quanto ao desenho dos processos de experimentação, a startup opta por desenvolver experimentos mais longos e exaustivos, ou seja, com maiores prazos de duração e maiores números de usuários e potenciais clientes experimentados, como forma de conhecer profundamente as necessidades, identificar variáveis potencialmente fatais para o negócio ou potencialmente impulsionadoras do sucesso (Blank, 2013; Ries, 2012; Sull, 2004) e verificar se os resultados do modelo de negócio são repetíveis e escaláveis (Blank \& Dorf, 2014), ainda que a velocidade na entrega do projeto final seja retardada.

Desta forma, o desenho do processo de experimentação baseado em hipóteses orientase inicialmente pelo teste do problema, seguido pelo teste da solução (Ries, 2012; Blank \& Dorf, 2014), a partir da identificação dos potenciais clientes (Ries, 2012; York \& Danes, 2014) e da estruturação das etapas baseadas no teste de funcionalidades em pequenos lotes (Ries, 2012). Assim, a seleção das hipóteses responde, essencialmente, a uma demanda latente identificada em uma experimentação anterior (Blank, 2013) ou ainda a identificação de uma hipótese que já foi validada e é oferecida em outros modelos de negócio.

A validação das hipóteses, por fim, utiliza o feedback do cliente como fonte principal e é acompanhada por métricas que analisam a experiência do usuário. Não se adota um parâmetro arbitrário de $n$ clientes para validação de um experimento, contudo, um mesmo experimento pode ser repetido de maneira mais exaustiva e em diferentes mercados-alvo.

\subsection{Caso 3}

A startup $\mathrm{C}$ ainda não realizou nenhuma validação mercadológica na prática e, consequentemente, um movimento de pivô da proposta de valor inicial, visto que a experimentação do MVP em desenvolvimento ainda não foi executada. Entretanto as hipóteses fundamentais para agregar valor aos clientes e usuários, bem como as métricas capazes de fornecer resultados reais de aprendizado, foram e ainda são desenvolvidas e testadas com especialistas da área. Isso demonstra o entendimento da startup de como os resultados devem ser interpretados (Euchner \& Ganguly, 2014) e caracteriza o que Ries (2012) denomina de contabilidade para a inovação. Isso poderá aumentar a garantia de que os resultados certos serão mensurados e avaliados, contribuindo para uma maior assertividade na validação ou rejeição das hipóteses do negócio (Frederiksen \& Brem, 2016; Ries, 2012).

Assim, a principal hipótese de valor criada foi resultado do conhecimento profundo dos clientes, dos fatores competitivos, técnicos e regulatórios do negócio, como também do conhecimento das variáveis que poderiam comprometer o negócio (Sull, 2004) obtido em experiências profissionais da carreira. Além disso, as experiências profissionais passadas permitiram ao fundador compreender o job to be done do problema a ser solucionado (Christensen et al., 2016) e, deste modo, também identificar as partes e as necessidades de cada parte (usuário e cliente) que integra o problema, desenvolvendo e apresentando valor a cada uma em específico. Portanto, a criação das hipóteses, até o momento, fundamenta-se na experiência do empreendedor (Sull, 2004), no conhecimento do profundo do problema (Christensen et al., 2016. Sull, 2004) e no conhecimento e feedback dos especialistas.

Neste caso percebe-se também que apesar de ainda não experimentada a solução, o desenho dos experimentos futuros já estão elaborados e se relacionam intimamente ao processo

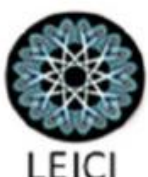


proposto por Ries (2012), uma vez que na visão do empreendedor este possibilita a startup reduzir riscos, minimizar perdas financeiras e flexibilizar uma possível necessidade de reconfiguração rápida (Blank, 2013; Ries, 2012; Teece et al., 2016). Ainda neste sentido, existe a identificação clara no desenho dos experimentos dos clientes e usuários e de como avaliar os resultados do experimento sob cada perspectiva, por meio de um questionário.

Por fim, para a validação das hipóteses foi desenvolvido, junto aos especialistas, um conjunto de aproximadamente 60 variáveis quantitativas que irão compor os questionários aplicados durante a experimentação. Para interpretação dessas variáveis serão utilizadas técnicas estatísticas apropriadas, de modo a reduzir o viés de julgamento do experimento (York \& Danes, 2014). Assim, somente depois da interpretação dos resultados estatísticos obtidos que investimentos adicionais serão buscados e comprometidos para dar início ao processo de oferta exponencial da oferta (Blank \& Dorf, 2014; Euchner \& Ganguly, 2014) ou de pivô (Blank, 2013; Ries, 2012).

\subsection{Caso 4}

Desde sua concepção a startup D utilizou-se de um pivô como um movimento de mudança estruturado para composição de uma nova hipótese estratégica fundamental a respeito da proposta de valor e da solução (Ries, 2012; Blank, 2013). Esse movimento foi resultado de uma avaliação e interpretação das informações obtidas em pesquisas informais utilizadas para reduzir o grau de ambiguidade e incerteza do negócio (Teece et al., 2016), a partir da identificação de variáveis que provavelmente comprometeriam o negócio (Sull, 2004; Blank \& Dorf, 2014).

Neste sentido, apesar da falta de experiência empreendedora do fundador na área de desenvolvimento do negócio em específico, a imaginatividade, enquanto uma habilidade que pode ser exercida e aprimorada em outras experiências empreendedoras para identificar e selecionar novas oportunidades de negócio (McMullen \& Kier, 2017), assim como o monitoramento constante das informações do setor é fundamental para a criação e seleção das hipóteses neste caso. Além disso, de modo a suprir a falta de contato com clientes potenciais fator esse restringido pela natureza ainda não legal da solução -, a empatia do empreendedor é uma técnica utilizada recorrente para identificar hipóteses de valor, que são impedidas de ser obtidas por meio da experimentação real.

Diante do contexto apresentado no caso, o experimento para validação da solução ainda não foi estruturalmente desenhado. Contudo um MVP inicial foi construído exclusivamente para captação de investimentos. Deste modo, depois dos investimentos captados e legalização da oferta é que um MVP próximo da solução final será apresentado ao mercado e de fato validado como consequência da resposta do mercado (Blank, 2013).

Assim, no geral, o caso demonstra um desenvolvimento mais distante da iteração constante com o cliente como forma de construção incremental da solução, diferentemente do proposto por Ries (2012) e Blank (2013) e mais baseado no conjunto de crenças que o empreendedor julga ser "verdadeiro" (Ries, 2012; Blank \& Dorf, 2014; York \& Danes, 2014) a partir da atribuição de sentido das informações que o mesmo tem acesso (Teece et al., 2016). Esse processo parece ser mais viável e conveniente ao negócio, uma vez que a regulamentação do mercado ainda não permite que a solução proposta seja experimentada, dificultando, portanto, o desenvolvimento do processo sob a perspectiva do customer development. 


\subsection{Análise cruzada}

No que se refere ao processo de criação das hipóteses, observa-se que elas são, em sua maioria, imaginadas e criadas a partir de experiências profissionais ou empreendedoras passadas e suportadas ora pelo conhecimento prévio das necessidades dos clientes e potenciais clientes, fatores competitivos, técnicos e regulatórios do negócio (Sull, 2004), ora pela capacidade de interpretação e atribuição de sentido das informações obtidas em relação ao negócio e ao setor (Sull, 2004; Teece et al., 2016). Outro importante aspecto refere-se ainda à capacidade de detecção generativa do empreendedor em identificar padrões e tendências sobre aspectos futuros diversos (Teece et al., 2016) e submetê-los a teste em seus modelos de negócio.

Além disso, a imaginatividade, enquanto uma habilidade cognitiva que combina a imaginação e o conhecimento tácito à capacidade de criar e aplicar a hipótese criada à ideia do empreendimento (McMullen \& Kier, 2017), também apresenta importante contribuição ao processo de construção e desenvolvimento das hipóteses. E neste aspecto, a utilização do job to be done (Christensen et al., 2016), ainda que de forma intuitiva e não lieterária, também revelase como uma importante técnica de suporte para identificação de problemas reais e construção de hipóteses. No entanto para que seja possível a liberação do fundador para essas atividades mais relacionadas ao processo de detecção e avaliação de oportunidades e, consequentemente, criação de hipóteses é essencial que a terceirização e descentralização das tarefas operacionais durante o desenvolvimento da solução seja estimulada, tornando o sistema mais flexível e mais rápido na apreensão das oportunidades (Teece et al., 2016).

Sequencialmente a criação das hipóteses, a seleção para teste segue, em sua maioria, um exame inicial da atratividade, escalabilidade e viabilidade sob a perspectiva das próprias experiências e aprendizados do empreendedor, bem como dos conhecimentos acumulados sobre o mercado, de modo a triar uma hipótese ou conjunto de hipóteses aparentemente mais promissor antes mesmo que uma experimentação real seja realizada (Sull, 2004). Ainda conforme observado, especialistas, como no caso 3 , ou a empatia, como no caso 4, podem ser também utilizados nesse processo de seleção.

Os MVPs, assim como proposto pelo método lean startup, são o principal meio utilizado para a experimentação das hipóteses junto aos clientes. Desta forma, eles são projetados para reduzir fontes de incerteza e responder a questões críticas para o sucesso do negócio, antes que recursos adicionais sejam comprometidos (Euchner \& Ganguly, 2014; Ries, 2012; Sull, 2004). No entanto apesar de utiliza-los como um meio comum para teste, o desenho do experimento em si varia conforme os casos, refletindo essencialmente o conjunto de aprendizados obtidos durante a sua trajetória (Teece et al., 1997, Teece et al., 2016).

Além disso, os desenhos experimentais respondem, em sua essência, também ao contexto do negócio. Entretanto é comum que o delineamento do teste seja orientado pelo teste inicial do problema e seguido pelo teste da hipótese de valor imaginada (Blank \& Dorf, 2014; Ries, 2012) e das hipóteses sequenciais de otimização em pequenos lotes (Ries, 2012). O caso da startup A, contudo, utiliza um desenho experimental baseado no teste simultâneo de $n$ hipóteses, diferenciando-se neste sentido da proposta do customer development.

Assim, o processo orientado por hipóteses nos casos estudados sustenta-se sobre as "intuições" iniciais dos empreendedores em relação ao seu modelo de negócio e se utiliza, no geral, do processo iterativo com clientes e usuários para aquisição do conhecimento para

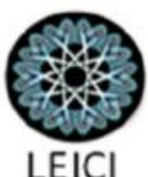


validação dos testes (Blank, 2013; Blank \& Dorf, 2014; Ries, 2012; York \& Danes, 2014). Cada hipótese é testada de maneira iterativa e analisada, fornecendo um conjunto de informações que se traduzem em uma decisão e um curso de ação futuros (Blank, 2013).

Deste modo, apesar de identificado como um processo de testes informal e, desta forma, propenso a erros e vieses de julgamento (York \& Danes, 2014), não foi identificado a presença eminente desses erros e vieses na validação das hipóteses nos casos analisados. Ao, contrário, de maneira prática, para reduzir esses riscos de comprometer a validação os casos desenvolvem e utilizam determinados rigores associados ao processo de experimentação, como o número de clientes e usuários consultados, o tempo de experimentação prolongado, experimentações intencionalmente repetidas em diferentes ambientes, além de métricas acionáveis (Ries, 2012) para a tomada de decisão. No caso 3, em específico, observa-se ainda uma maior aproximação de técnicas e métodos estatísticos para suporte a validação das hipóteses. E no caso 4, uma tentativa de se colocar em terceira pessoa (empatia).

\section{Conclusão}

As observações obtidas a partir dos quatros diferentes casos analisados neste estudo permitem concluir que a abordagem do customer development fornece aos empreendedores um guia ou panorama de orientação flexível o suficiente para ser adaptado ao contexto no qual o negócio desenvolve-se, bem como aos recursos e condições disponíveis para o desenho e execução do processo. Assim, a abordagem organiza - sem, contudo, restringir as adaptações necessárias ou convenientes - o processo de desenvolvimento do negócio, orientando as ações empreendedoras e sistematizando esse processo, por meio da criação e experimentação das hipóteses, e utilização do feedback dos clientes como a principal forma para descobrir se as suposições de negócio imaginadas estão corretas ou não. Em termos gerais, a abordagem é, portanto, utilizada com proposta, salvo algumas ressalvas e adaptações.

Assim, apesar de um poder adaptativo os resultados parecem sugerir, contudo, que para negócios que desenvolvem soluções mais disruptivas, ou ainda em mercados altamente regulamentados, esse desenvolvimento orientado por hipóteses pode não ser perfeitamente estruturado quando comparado ao processo proposto pela abordagem, uma vez que a presença de determinadas limitações associadas ao contexto do negócio são capazes de dificultar uma ou mais etapas do processo. Como, por exemplo, o desenvolvimento do MVP e a possibilidade de iteração com os usuários e potenciais clientes, o que reflete num desenvolvimento mais dependente da empatia e da capacidade de imaginação e abdução do empreendedor para conduzir o desenvolvimento do negócio. Neste aspecto, estudos futuros sobre a aplicação da abordagem nesses contextos específicos podem contribuir para a melhor compreensão do indício e consequente adaptação da abordagem, caso necessário.

Além disso, apesar de empiricamente comprovada a eficiência da abordagem, outras observações presentes neste estudo sugerem algumas contribuições para o aprimoramento e completude da abordagem, visto sua proposta de fornecer um processo para a criação de negócios com menores níveis de incerteza e maiores probabilidades de sucesso. Dentre elas, o exame inicial da atratividade, escalabilidade e viabilidade das hipóteses imaginadas antes da experimentação real da hipótese em si, utilizando-se das próprias experiências e aprendizados, bem como dos conhecimentos acumulados sobre o mercado e a aplicação do job to be done sobre o problema a ser solucionado. Este, por último, pode ser uma valiosa técnica de suporte

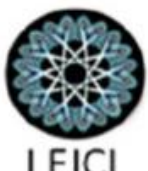


para a construção de um MVP menos propenso a erros de teste, sejam eles técnicos ou de valor, e de uma experimentação mais próxima a uma experiência de consumo e não apenas a um meio de teste para obtenção de informações.

Outros dois aspectos importantes observados são: (i) ao se focar no que se denomina popularmente como "ouvir os clientes", a abordagem parece diminuir ou até mesmo negligenciar as capacidades empreendedoras de criar e integrar hipóteses de negócio originadas pela imaginativadade e capacidade generativa do empreendedor. No entanto, essa constatação não segure que o desenvolvimento da solução deva negligenciar a participação dos clientes. Ela apenas evidencia uma fonte igualmente capaz de oferecer valor e atender necessidades ocultas de forma inovativa, assim como criar e desenvolver hipóteses; (ii) quanto à validação das hipóteses, apesar de essencialmente informal, observa-se que como resposta a aprendizados empreendedores passados, rigores ainda que não estatísticos são adotados, na tentativa de aumentar a confiabilidade da interpretação dos resultados. Supõe-se, deste modo, que empreendedores iniciais podem ser mais propensos a esses erros. Isso evidencia outro aspecto a ser mais desenvolvido na abordagem em questão.

Por fim, as principais limitações deste estudo referem-se ao número amostral analisado e ao método de pesquisa seccional utilizado. Por isso, além das propostas de estudo futuras já apresentadas sugere-se também a aplicação de pesquisas de natureza similar com abordagem quantitativa ou ainda estudos qualitativos longitudinais.

\section{Referências}

Al-Ashaab, A., Golob, M., Urrutia, U. A., Gourdin, M., Petritsch, C., Summers, M., \& ElNounu, A. (2016). Development and application of lean product development performance measurement tool. International Journal of Computer Integrated Manufacturing, 29(3), 342354.

Bardin, L. (2010). Análise de conteúdo. Lisboa, Portugal: Edições, 70.

Bieraugel, M. (2015). Managing library innovation using the lean startup method. Library Management, 36(4/5), 351-361.

Blank, S. (2013). Why the lean start-up changes everything. Harvard business review, 91(5), 63-72.

Blank, S., \& Dorf, B. (2014). Startup: manual do empreendedor. Alta Books Editora.

Christensen, C. M., Hall, T., Dillon, K., \& Duncan, D. S. (2016). Know your customers “jobs to be done". Harvard Business Review, 9, 54-62.

Cooper, D. R., \& Schindler, P. S. (2016). Métodos de Pesquisa em Administração-12a Edição. McGraw Hill Brasil.

Crews, C. (2016). The Focus Question and Business Model Innovation. Research-Technology Management, 59(3), 62-64.

Edison, H. (2015, December). A conceptual framework of lean startup enabled internal corporate venture. In International Conference on Product-Focused Software Process Improvement (pp. 607-613). Springer International Publishing.

Edison, H., Smørsgård, N. M., Wang, X., \& Abrahamsson, P. (2018). Lean Internal Startups for Software Product Innovation in Large Companies: Enablers and Inhibitors. Journal of Systems and Software, 135, 69-87. 
Eisenmann, T. R., Ries, E., \& Dillard, S. (2013). Hypothesis-driven entrepreneurship: The lean startup.

Euchner, J., \& Ganguly, A. (2014). Business model innovation in practice. ResearchTechnology Management, 57(6), 33-39.

Frederiksen, D. L., \& Brem, A. (2016). How do entrepreneurs think they create value? A scientific reflection of Eric Ries' Lean Startup approach. International Entrepreneurship and Management Journal, 13(1), 169-189.

Hair, J., Babin, B., Money, A., \& Samouel, P. (2005). Fundamentos de métodos de pesquisa em administração. Bookman Companhia Ed.

Janeway, W. H. (2012). Doing capitalism in the innovation economy: markets, speculation and the state. Cambridge University Press.

McMullen, J. S., \& Kier, A. S. (2017). You don't have to be an entrepreneur to be entrepreneurial: The unique role of imaginativeness in new venture ideation. Business Horizons, 60(4), 455-462.

Ries, E. (2012). A startup enxuta: como os empreendedores atuais utilizam a inovação para criar empresas extretamente bem-sucedidas. Leya.

Sobek II, D. K., Ward, A. C., \& Liker, J. K. (1999). Toyota's principles of set-based concurrent engineering. Sloan management review, 40(2), 67.

Sosna, M., Trevinyo-Rodríguez, R. N., \& Velamuri, S. R. (2010). Business model innovation through trial-and-error learning: The Naturhouse case. Long range planning, 43(2), 383-407.

Sull, D. N. (2004). Disciplined entrepreneurship. MIT Sloan Management Review, 46(1), 71.

Tanev, S. (2017). Is There a Lean Future for Global Startups?. Technology Innovation Management Review, 7(5).

Teece, D. J., Pisano, G., \& Shuen, A. (1997). Dynamic capabilities and strategic management. Strategic management journal, 509-533.

Teece, D., Peteraf, M., \& Leih, S. (2016). Dynamic capabilities and organizational agility. California Management Review, 58(4), 13-35.

Yin, R. K. (2015). Estudo de Caso: Planejamento e Métodos. Bookman editora.

York, J. L., \& Danes, J. E. (2014). Customer development, innovation, and decision-making biases in the lean startup. Journal of Small Business Strategy, 24(2), 21-39.

Ward, A. C., \& Sobek II, D. K. (2014). Lean product and process development. Lean Enterprise Institute. 